\title{
Prospects of model-based fault diagnostics for dynamic traffic control systems
} on freeways

\author{
Thorsten Neumann \\ Institute of Transportation Systems, German Aerospace Center (DLR), Germany. \\ E-mail: thorsten.neumann@dlr.de
}

\author{
Anja Estel \\ Traffic Management Department, Straßen.NRW - Traffic Center, Germany. \\ E-mail: anja.estel@strassen.nrw.de
}

\begin{abstract}
Dynamic traffic control systems on freeways are highly complex facilities used for optimizing traffic flow and increasing traffic safety by, for instance, harmonizing speeds and displaying warnings to the road users. At the same time, the decentralized design of such systems comprising many distributed and inter-connected hardware and software components makes them costly to maintain in practice. Although there are several monitoring tools available, fault diagnostics still is an effortful (manual) process involving experienced staff in the traffic control center and in the field. Model-based support including the automatic generation of case-specific troubleshooting strategies depending on observed failures and evidences provide the chance to considerably increase the efficiency of fault management in this context. Bayesian networks as an established probabilistic framework for diagnostic reasoning in complex scenarios is discussed as a suitable model approach to meet these mentioned challenges. The general concept includes a transparent and human-interpretable deduction of diagnostic results as well as a high compatibility with current management processes in the traffic control center (e.g., ticketing). Not least, available system information and domain knowledge as already collected by common failure mode and effect analyses or similar approaches will support the creation of a valid diagnostic model in terms of a Bayesian network what is not possible in such an easy way for other popular modelling approaches as, for instance, neural networks.
\end{abstract}

Keywords: Road traffic, dynamic traffic control, fault diagnostics, Bayesian networks, PHM, maintenance.

\section{Introduction}

Dynamic traffic control systems are important technical assets of the road infrastructure with regard to the efficiency and safety of traffic on highly utilized freeways (cf. FGSV, 2008). Based on distributed system architectures, they typically consist of numerous local sensors for measuring traffic flow and environmental conditions (cf. BMVI, 2018). Centralized and decentralized hardware and software components are responsible for data processing (including rule-based automated traffic control) and data communication. Human interaction in terms of manual control (as, for instance, in case of accident warnings) as well as continuous system monitoring is realized by operators in a traffic control center. Finally, from the viewpoint of the road users, the most visible components of such traffic control systems are the dynamic traffic signs used for displaying warnings (e.g., congestion, wet or icy road conditions, or accidents), speed limits, and possible restrictions on overtaking.

Obviously, dynamic traffic control systems as described above are highly complex assets and thus difficult and expensive to maintain. In fact, fault identification often is an effortful manual process currently realized more or less systematically by experienced operators and maintenance engineers in the traffic control center and in the field (cf. Section 2). Modelbased tools for automatic failure detection and diagnosis (i.e., identification of failure reasons) such as Bayesian networks (cf. Section 3) therefore provide the chance to significantly improve the efficiency of current maintenance processes.

The present contribution discusses these potentials from a scientific as well as a practitioner's point of view (cf. Section 3) including a critical review of current maintenance strategies (cf. Section 2) and previous work on failure diagnostics for dynamic traffic control systems and comparable technical assets (cf. Section 3.2). The paper ends with some conclusions in Section 4.

\section{Current Practices}

The NRW Traffic Center plans, builds and operates all collective traffic control systems as part of the Intelligent Transport Systems (ITS) infrastructure on the motorways in the German federal state of North Rhine-Westphalia. These include line control systems, systems for

Proceedings of the 30th European Safety and Reliability Conference and the 15th Probabilistic Safety Assessment and Management Conference.

Edited by Piero Baraldi, Francesco Di Maio and Enrico Zio

Copyright (C) 2020 by ESREL2020 PSAM 15 Organizers. Published by Research Publishing, Singapore

ISBN: 981-973-0000-00-0 :: doi: 10.3850/981-973-0000-00-0 esrel2020psam15-paper 


\section{Thorsten Neumann and Anja Estel}

temporary release of hard shoulder, ramp metering systems as well as network control systems. Traffic control depending on the current traffic situation is partly realized as automatic process via the sub-centers, but some functions are also manually controlled by the operators in the control room of the traffic control center. The aim of traffic control is to increase traffic safety and to harmonize the traffic flow in order to reduce congestion. To achieve these goals comprehensively, a high availability of all related components in $24 / 7$ operation must be guaranteed.

The actual traffic control as one core process of the traffic control center must be separated from the technical operation of the system components and their maintenance. In Germany, the ITS infrastructure on the motorways fall under the so-called Act on the Federal Office for Information Technology (BSIG) as critical infrastructure. In this context, the systems must demonstrably fulfil special requirements regarding availability and integrity. But also from the quality management point of view, the technical operation of the systems is of great importance. As described in Neumann et al. (2017), the "maintenance and repair" process has a particularly high influence on failure rates and error propagation and thus on the quality and effectiveness of traffic management measures, in addition to a number of other processes, that are outlined in Estel and Rose (2021).

Numerous components are required which together form the overall traffic control system. Figure 1, for example, shows the system "line control" with its main components. The measuring sections are used to monitor the traffic situation, and this information is passed on to the sub-control center via dedicated roadside stations. There, speed limits and warnings adapted to the traffic situation are selected by specialized algorithms which are then send to the displays at the corresponding cross sections on the road. At the same time, traffic data and information about the displayed warnings et cetera are transmitted via the remote bus to the traffic control center, where they are stored and analyzed. This includes forwarding them via various networks to the control room for being displayed to the operators.

In the case of construction sites as well as unforeseeable events like accidents, the operators have to override displays and information to the road users as generated by the automatic algorithms of the sub-center, i.e. send control commands from the operating computer through various communication networks back to the sub-center and to the roadside station. The variable message signs at the traffic sign gantries must then quickly and clearly display the desired traffic signs in the required brightness.

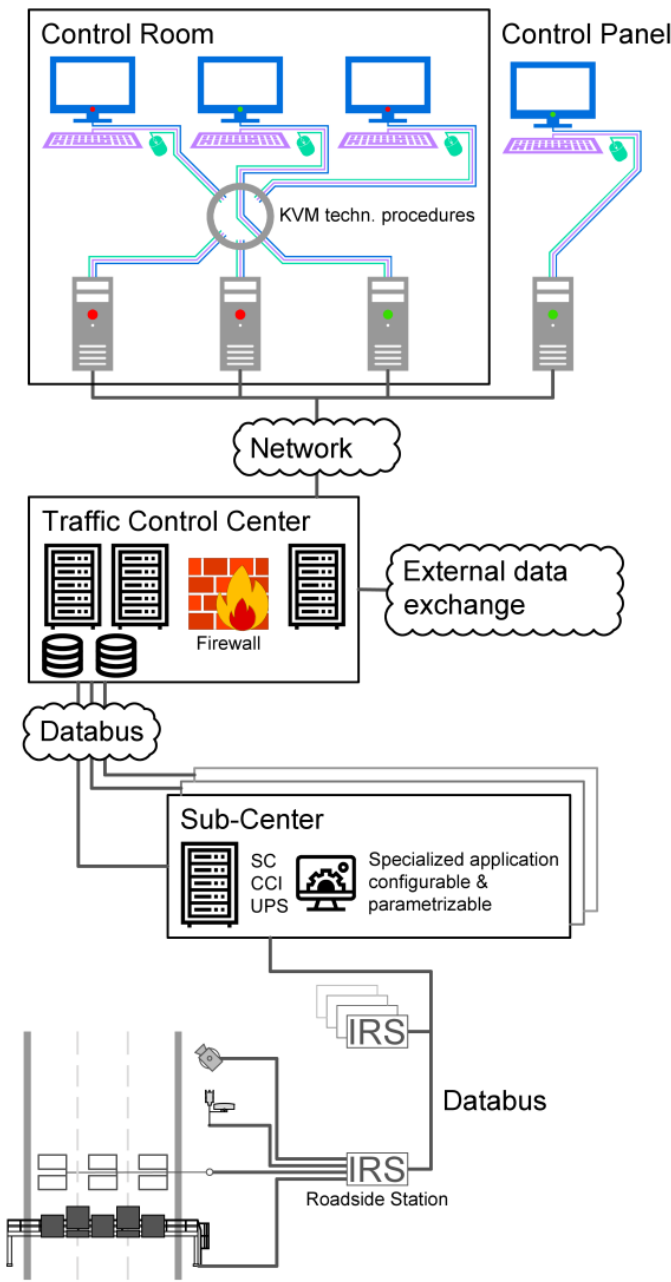

Figure 1: System components of a line control system.

Only if all components work reliably, all functions can be fully executed and the full economic benefit in terms of traffic safety and traffic flow can be achieved. For this reason, with few exceptions, all components are kept in as fail-safe operation as possible through extensive maintenance and repair contracts. These contracts not only cover technical components (roadside stations, traffic sign transmitters, cameras ...) or computer hardware (operating computers, servers, routers, switches ...), but also the specialist applications, operating systems and firewalls used (e.g. through extensive patch management). Maintenance in the sense of proactive support is carried out on the IT side by means of internal and external operational monitoring, while the track-side 
components and sub-centers are inspected on site every six months. If a component fails, repair measures are initiated. The contracts provide fixed reaction times. Service level agreements have been concluded for some, but not all individual components.

However, in the malfunction event, it is often challenging to isolate and identify the cause of failure or the component actually affected which hinders the fault from being eliminated as quickly as possible. Malfunctions can be detected in the control room, on the line or by service providers and must then lead to the triggering of the fault management support process. Due to the complexity of the overall system and the different responsibilities for individual components, the fault management process has also been time-consuming up to now. Although there are several operational monitoring tools now (cf. Estel and von der Ruhren, 2013), especially in the area of IT components and networks, which can help to locate the source of error, it is not possible to consider all components with these tools. Nor can the direction of cause and effect be clarified beyond doubt. There is also a lack of meaningful documentation on past faults and their causes (incl. protocols of the elimination), which could be helpful in current fault situations in terms of defining suitable troubleshooting strategies.

For the purpose of improving the related processes, the existing practices were analyzed and essential difficulties (pain points) were identified. In addition to the lack of documentation and insufficient lessons learned with all parties involved, the ticket system used up to that point was also identified as insufficient with regard to the requirements. Organizational issues such as responsibilities and lack of feedback on fault events were also mentioned. Due to missing content-supporting documentation of previous trouble shootings and experiences (e.g. error trees), every new fault leads to a new search. This could be improved by a regular analysis of comprehensively documented fault rectifications.

As a result of this analysis, the essential contents of fault management were derived using the SIPOC method (SIPOC = suppliers, inputs, process, outputs, and customers). A new ticket system (open source) is currently being introduced, which has an interface to the existing Configuration Management Database (CMDB) which allows access as well as management of so-called Configuration Items (CI), such as servers, computers, IT services and network components, but also ITS roadside elements. This means that all components that could be affected by a fault are already known, as well as the associated system components or communication paths. Within the framework of an internal working group, cause-effect relationships are worked out on the basis of the employees' wealth of experience using methods for problem solving (e.g. Ishikawa diagrams, FMEA). Probabilities and frequencies are derived in order to use support tools more effectively in the future within the framework of fault management, and also in order to change the sequence of the components to be tested depending on the fault pattern. The overall aim is to speed up the identification of causes of error in the case of failure.

\section{Improved Fault Diagnostics using Bayesian Networks}

The current practices (see Section 2) show that systematic tools for fault diagnostics provide the chance to significantly reduce the (manual) efforts in the context of fault management of dynamic traffic control systems on freeways. In this regard, models should not only represent the qualitative cause-effect relations but should also be able to provide quantitative estimates of fault probabilities and to automatically propose dedicated troubleshooting strategies depending on given evidences on failures and states of the system components.

Bayesian networks as a well-established mathematical approach for diagnostic reasoning provide an ideal framework with regard to the mentioned tasks (cf. Fenton and Neil, 2013). Moreover, the process of designing Bayesian networks based on expert knowledge can be seen in close relation to other common (qualitative) approaches (e.g., FMEA) for eliciting causes and effects in technical systems. In fact, some extended methods based on failure nets and/or probabilistic FMEA (cf. Kaiser and Rauschenbach, 2015) can directly be used for systematically creating Bayesian networks from such knowledge (cf. Rauschenbach and Nuffer, 2019).

Not least, Bayesian networks have the favorable property that they provide a highly transparent mathematical representation of even complex (technical) systems. That is, in contrast to other popular approaches such as neural networks, the diagnostic reasoning process of Bayesian networks can usually be validated directly by humans via interpreting not only final but also intermediate results step by step. Clearly, this kind of readability of the model is a big advantage with regard to practical applications. Indeed, the acceptance of diagnostic tools often depends on whether they can intuitively be understood by technicians from the respective domain as was mentioned by Silmon and Roberts (2010) with regard to railway switches, for instance. 


\section{Thorsten Neumann and Anja Estel}

Another important aspect, also mentioned by Silmon and Roberts (2010), is that the diagnostic reasoning and the computations needed for that can be executed automatically. With numerous exact and approximate algorithms available for solving this task, Bayesian networks completely fulfil this requirement. In fact, various existing software tools and libraries for editing and computing Bayesian networks provide even additional support for deeper studies on the implemented models including sensitivity analyses (Coupé and van der Gaag, 2002) or algorithms for parameter learning (Mahjoub and Kalti, 2011). That is, the integration of Bayesian diagnostic models with other software components of the fault management process such as the CMDB or the ticket system mentioned in Section 2 is not an insurmountable problem but just a matter of technical implementation of interfaces and workflows.

\subsection{Theoretical approach}

Bayesian networks are a special kind of probabilistic graphical models (Koller and Friedman, 2009), which allow the visual representation and compact coding of complex stochastic dependencies between different random variables (= nodes) with any number of (discrete) states. With regard to a traffic control system as in Figure 1, these random variables could be defined by the components and subcomponents of the system together with their possible failure states and faults. The nodes relevant to the problem are arranged then in the form of a directed, acyclic graph (cf. Figure 2). Edges represent the (usually causal) influence of a node on its immediate successors (= child nodes). Moreover, the dependency structure between more distant nodes complies with the graph-theoretical principle of d-separation or dconnectivity (Charniak, 1991).

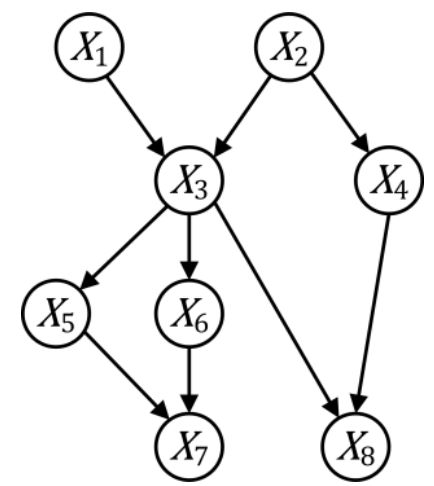

Figure 2: Bayesian network (Example)
This enables complex forms of model-based reasoning in which knowledge (= evidence) about the state of individual nodes can be used to efficiently calculate the resulting (conditional) state probabilities of all other nodes in the network. Causal reasoning happens when knowledge about the state of a node $X_{i}$ affects the state probabilities of one or more (not necessarily immediate) successors $X_{j}$. Conversely, diagnostic reasoning means that evidences for a given node affects the probabilities of the parent or further upstream ancestor nodes. Finally, intercausal reasoning is of particular interest when there are two or more possible causes for a given observed (direct or indirect) effect. If, for example, one of these possible causes $\left(X_{i}\right)$ can be eliminated as the reason for the observed effect, the probabilities of the other causes usually increase. Conversely, the probability of the other causes decreases if $X_{i}$ (at least in part) can be identified as the actual trigger for the given effect. In the literature, this phenomenon is also referred to as "Explaining Away" (Koller and Friedman, 2009).

Besides the fundamental stochastic dependencies between the random variables of the Bayesian network, which are almost completely coded by the structure of the underlying graph, the actual quantification of the mutual influence between the nodes of the network is represented by local conditional probability distributions of the form $\mathrm{P}\left(X_{i} \mid \mathrm{Pa}\left(X_{i}\right)\right)$, where $\operatorname{Pa}\left(X_{i}\right)$ is the set of parent nodes of the node $X_{i}$. The full definition of a Bayesian network thus requires the construction of the underlying graph and, in a second step, the determination of the local (conditional) probability distributions for each single node (= parametrization).

Once all this is done, the Bayesian network can be used for automatically computing the marginal and conditional probabilities of all node states depending on any type of evidence in terms of exact (or even uncertain) knowledge about the true state of some of the nodes in the network. That is, given a certain malfunction of the modelled technical system, the Bayesian network consistently yields suitable estimates for the probabilities of all possible faults that may have caused the observed malfunction. Notably, it is even possible to iteratively feed the network with further evidence whenever there are new findings concerning some of the nodes, e.g., because of additionally performed tests. Thus, Bayesian networks systematically help to localize the true cause of failure by supporting the probabilistic reasoning process when analyzing the available evidences with regard to the given malfunction. 
Even more, Bayesian networks do not only mimic and automatize human reasoning under uncertainty but can also be used for deriving optimal troubleshooting strategies (cf. Huang et al., 2015). That is, by automatically analyzing the sensitivities in the model, for instance, it is possible in each step to detect those nodes in the network where additional knowledge about their states yields the most information with regard to reducing the uncertainty about the actual cause of failure. In other words, possibly weighted by relevant costs and/or efforts, the Bayesian network could tell the user directly what to do next given any specific error pattern, i.e., what component should be checked or which additional diagnostic test should be performed. Needless to say, this approach essentially helps to structure and harmonize the process of fault identification and finally makes it independent of the individual experience and knowledge of the operator or maintenance engineer on duty. Nonetheless, the underlying model has to be validated, of course, involving experienced professionals as well as possibly available documentation of previous failures.

\subsection{Previous research}

Bayesian networks have been used in many technical disciplines so far. Cai et al. (2017) provide an extensive review of recent applications for energy systems, manufacturing systems and others. In addition to that, clinical diagnostics is a well-established field where Bayesian networks have been developed and applied successfully over the years (Sierra et al., 2000). In fact, some of the most famous examples of Bayesian networks such as the Pathfinder model (Heckerman and Nathwani, 1992) have arisen from this field.

With regard to transportation systems, Bayesian networks have been proposed for assessing road traffic safety (Grande et al., 2017), detecting travel modes (Xiao et al., 2015), or modeling travellers' satisfaction with railway transport (Perucca and Salini, 2016), for instance. In particular, recent ideas in context of fault diagnostics for railway switches (Neumann et al., 2019) are very similar to what is proposed here to be done for dynamic traffic control systems on motorways.

Not least, Neumann et al. (2017) described a Bayesian network trying to represent how faults or deficiencies from planning to operation affect the functionality of dynamic traffic control systems. Using a structured FMEA, the authors derived a functional model where all relevant processes and elements of a hypothetical dynamic traffic control system were listed in functional relation to each other. The goal here was to identify the most crucial technical and/or organizational processes in the life cycle of such facilities in order to improve the quality of traffic control by optimizing these processes as part of a systematic quality management (cf. Estel and Rose, 2021). In this regard, Neumann et al. (2017) found that "maintenance and repair", for instance, is one the most crucial processes when operating a traffic control system over the years. However, due to structural limitations - such as simplified error propagation via aggregated (instead of detailed) fault modes - the model from Neumann et al. (2017) did not allow for proper diagnostics in terms of reliably identifying specific causes of error in case of given observed failures. In fact, the respective Bayesian network was shown to be a mostly qualitative rather than a precise quantitative model.

Consequently, there still is a strong need for systematic and automated tool support with regard to fault diagnostics of traffic control systems in order to further improve the maintenance process and thus to increase reliability and availability of the relevant assets.

\subsection{Model implementation}

Even if there is no real implementation yet, Figure 3 shows the draft layout of a possible Bayesian network that could apply to the proposed context. As can be seen, the nodes are closely related to the real components of a dynamic traffic control system as depicted in Figure 1. The red edge labels, by the way, refer to the main functionality of each node with regard to its respective child. That is, faults related to this node-specific functionality can be expected to directly infer the proper working of the subsequent components.

The roadside station, for instance, will usually not be able to provide suitably aggregated data in case of major defects of the connected sensors even if there might be algorithms for correcting or interpolating missing and/or erroneous data. Furthermore, the sub-center requires the unimpeded data communication via a local data bus in order to receive (correct) data from the roadside station. And, the control panels in the control room (cf. Figure 1) depend on the network connection to the data management system of the traffic control center as well as on the reliability of the data management itself.

Finally, displaying variable traffic signs and warnings adequately to traffic and environmental conditions is possible only if there are correct switching commands from the roadside station together with well-performing algorithmic control at the sub-center and/or accurate manual control via the control panels. Of course, all relevant communication lines (i.e. network and/or data buses) have to be available, too. 


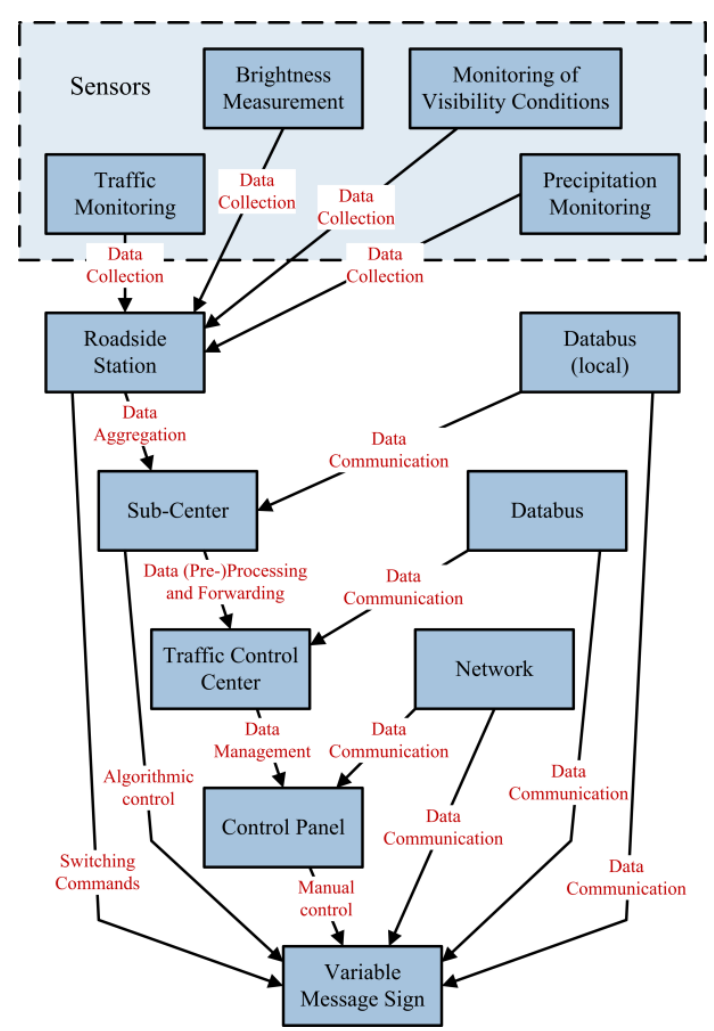

Figure 3: Possible basic structure of the proposed Bayesian network (Draft)

Needless to say, the model from Figure 3 is certainly not complete. Important components such as power supply or others are still missing obviously. Moreover, fault states per node including their mutual dependencies need to be described and quantified in detail. The corresponding concretization and further development including possible revisions of the proposed draft model from Figure 3 will be part of future work.

\subsection{Practical benefits}

Due to the complexity of the overall structure of traffic control systems and the numerous individual causes of failure per component, a targeted probabilistic troubleshooting strategy based on the concept as outlined in Section 3.1 would definitely support the fault management process. By limiting and prioritizing the possible causes of error for given error patterns and by being able to further reduce the number of error sources by entering further known evidence, e.g. tests that have already been carried out, the process of finding the true cause of failure can be simplified and employees can be relieved from testing less probable sources of error.
The benefit becomes even larger if the tools for optimizing the case-specific troubleshooting strategy are directly linked to the ticket system of the fault management (cf. Section 2). In this regard, the ticket process between the persons responsible for the various components could be oriented towards the probabilities computed by the (Bayesian) diagnostic model and, depending on the fault pattern and already excluded causes of error, could be targeted towards the most probable faults. Another argument in favor of such integration into the ticket system is that fault processing can be carried out then by technical employees without the need for understanding the complex mathematical background of the diagnostic model - as the one proposed above - in detail.

Note that, as a first step and instead of a fully automated integration, the tool could of course also be placed with a trained first level support who manually determines the most probable causes using the model before forwarding the ticket accordingly to the maintenance staff. Therefore, even without larger efforts for the technical integration (i.e., without detailed implementation of software interfaces and automated workflows), benefits resulting from the application of the diagnostic model can be realized within the given ticket system.

On the long term, it could then be worked on to continuously and automatically update the parameters and inherent probabilities of the diagnostic model, which will initially be based on the experience of the employees, by systematically analyzing future failures, their fault patterns and causes, and thus to allow the model to make increasingly reliable statements. Needless to say, this definitely is a future second step from now on and requires establishing a systematic feedback about true causes of failure first including standardized processes for their documentation by the maintenance staff. In this case, a full technical integration with the official ticketing process would be very helpful, too.

\section{Conclusion}

As discussed in previous sections, the creation and application of a comprehensive (probabilistic) diagnostic model (cf. Section 3) for the complex cause-and-effect relations within the overall technical structure of a traffic control system have the potential to significantly simplify the process of fault management in the traffic control center including a speed-up of fault diagnosis and the avoidance of parallel work. That is, by applying such a model for automatically identifying the most probable failure reasons in case of malfunctions of the system and deriving optimal troubleshooting strategies based on current evidences, the 
efficiency of fault management is expected to increase. Disturbed traffic control systems or components can be repaired more quickly and thus economic damage in the sense of unrealized benefits with regard to traffic safety and traffic flow is reduced.

In this context, the next step will be to collect and transform already compiled expert knowledge about traffic control systems (cf. Section 2) in such a way that it allows to create a valid Bayesian network for a given (real) facility from that. Approaches for model and parameter elicitation such as described by Rauschenbach and Nuffer (2019) as well as experiences from previous research (e.g., Neumann et al., 2017) are expected to be very helpful in this regard. Once the model is calibrated and validated, the technical integration into the operational processes of the traffic control center will be an important milestone with regard to optimizing the fault management of the connected traffic control systems. Further studies could then try to expand the diagnostic model by dynamic aspects in such a way that it does not only allow for identifying existing faults but also to predict future failures based on available data and evidences where possible. This could facilitate even further improvements of the related maintenance processes by a shift towards more predictive instead of currently mostly corrective strategies.

\section{References}

BMVI (2018). Merkblatt für Verkehrsrechnerzentralen und Bundesanstalt für Straßenwesen die Ausstattung von Unterzentralen. Ausgabe 2018, Berlin, Germany.

Cai, B., Huang, L., and Min, X. (2017). Bayesian Networks in Fault Diagnosis. IEEE Trans. Ind. Inf. 13(5), 2227-2240.

Charniak, E. (1991). Bayesian Networks without Tears. AI Magazine, Winter 1991, 50-63.

Coupé, V.M.H., van der Gaag, L.C. (2002). Properties of sensitivity analysis of Bayesian belief networks. Annals of Mathematics and Artificial Intelligence 36, 323-356.

Estel, A., and Rose, M. (2021). Aufbau und Betrieb eines Qualitätsmanagements - Herausforderungen in der Praxis der Verkehrszentrale NordrheinWestfalen. In: HEUREKA 20, April, 13-14, 2021, Stuttgart, Germany.

Estel, A. and von der Ruhren, S. (2013). Traffic IQ: Pilotprojekt Informationsqualität im Verkehrswesen; Schlussbericht Teilvorhaben Straßen.NRW. Gelsenkirchen, Germany.

Fenton, N., and Neil, M. (2013). Risk Assessment and Decision Analysis with Bayesian Networks. CRC Press, Boca Raton.

FGSV (2008). Hinweise zur Wirksamkeitsschätzung und Wirksamkeitsberechnung von
Verkehrsbeeinflussungsanlagen. Heft 311, Köln, Germany.

Grande, Z., Castillo, E., Mora, E., and Lo, H.K. (2017). Highway and Road Probabilistic Safety Assessment Based on Bayesian Network Models. Computer-Aided Civil and Infrastructure Engineering 32(5), 379-396.

Heckerman, D.E., Nathwani, B.N. (1992). Toward normative expert systems: Part II. Probabilitybased representations for efficient knowledge acquisition and inference. Methods of Information in Medicine 31(2), 106-116.

Huang, Y., Wang, Y., and Zhang, R. (2015). Fault Troubleshooting Using Bayesian Network and Multicriteria Decision Analysis. Advances in Mechanical Engineering 6(5), 282013.

Kaiser, B., and Rauschenbach, M. (2015). Probabilistic Extension of Failure Net Based FMEA. In: ESREL 2015, September, 7-10, 2015, Zurich, Switzerland.

Koller, D., Friedman, N. (2009). Probabilistic Graphical Models - Principles and Techniques. MIT Press, Cambridge.

Mahjoub, M.A., and Kalti, K. (2011) Software Comparison Dealing with Bayesian Networks. In: Liu D., Zhang H., Polycarpou M., Alippi C., and $\mathrm{He}$ H. (eds.). Advances in Neural Networks - ISNN 2011. Lecture Notes in Computer Science, vol. 6677. Springer, Berlin, Heidelberg.

Neumann, T., Narezo Guzmán, D., and Groos, J.C. (2019). Transparent failure diagnostics for railway switches using Bayesian networks. Signalling + Datacommunication (111), 12/2019, 23-31.

Neumann, T., Schwietering, C., Fazekas, A., Jakobs, E., and Volkenhoff, T. (2017). Probabilistische Modellierung der Fehlerfortpflanzung im Lebenszyklus von Streckenbeeinflussungsanlagen. In: HEUREKA '17, March 22-23, 2017, Stuttgart, Germany.

Perucca, G., and Salini, S. (2016). Travellers' Satisfaction with Railway Transport: A Bayesian Network Approach. Quality Technology \& Quantitative Management 11(1), 71-84.

Rauschenbach, M., and Nuffer, J. (2019). Quantitative FMEA and Functional Safety Metrics Evaluation in Bayesian Networks. In: ESREL 2019, September, 22-26, 2019, Hannover, Germany.

Sierra, B., Inza, I., and Larrañaga P. (2000) Medical Bayes Networks. In: Brause, R.W., and Hanisch, E. (eds.). Medical Data Analysis. ISMDA 2000. Lecture Notes in Computer Science, vol. 1933. Springer, Berlin, Heidelberg.

Silmon, J.A., and Roberts, C. (2010). Improving railway switch system reliability with innovative condition monitoring algorithms. Proc IMechE Part F: Journal of Rail and Rapid Transit 224(4), 293-302.

Xiao, G., Juan, Z., and Zhang, C. (2015). Travel mode detection based on GPS track data and Bayesian networks. Computers, Environment and Urban Systems 54, 14-22. 